\title{
An old elasticity problem in a unilateral setting
}

\section{J. DUNDURS}

Civil Engineering, Northwestern University, Evanston, Illinois 60201, USA

\section{MARIA COMNINOU}

Civil Engineering, The University of Michigan, Ann Arbor, Michigan 48109, USA

(Received August 18, 1980)

\section{ABSTRACT}

A limiting case of the Michell problem involving an elastic wedge is the unbounded solid with a semi-infinite cut, the tip of which is subjected to a concentrated force. For the limiting case, the classical solution leads to overlapping of material whenever the component of the force along the axis of symmetry is directed away from the cut, and the problem must be solved anew using unilateral boundary conditions. The required mathematics is simple, and the subject is suitable for classroom discussion. Two examples are solved explicitly, and additional exercise problems are suggested.

\section{Introduction}

Elasticity solutions involving unilateral boundary conditions tend to be complicated and most are not well suited for classroom discussion or student exercises. A simple situation illustrating many aspects of boundary conditions with inequalities, and particularly those associated with frictional slip, evolves, however, from the Michell problem of a wedge subjected to a concentrated force at its vertex [1]. A limiting case of the wedge is the unbounded solid with a semi-infinite cut or crack. The simplicity of this problem in a unilateral formulation is due to the fact that it contains no characteristic length independent of the applied load $P$. Consequently, since the contact is receding and therefore independent of the level of the applied load [2], it is clear that the two sides of the cut are either separated or in contact everywhere. Moreover, the two faces must either stick or slip everywhere in case of contact.

\section{Boundary conditions and form of solution}

Consider the unbounded solid with a straight semi-infinite cut. The tip of the cut is subjected to a concentrated force of magnitude $P$. The coordinate system is placed in relation to the solid as shown in Fig. 1. 


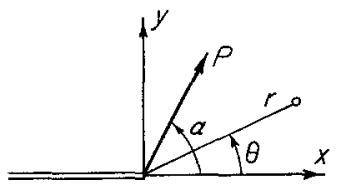

Figure 1

The quantities entering the boundary conditions on the cut are the normal tractions

$$
N=\sigma_{\theta \theta}(r, \pm \pi),
$$

the shearing tractions

$$
S=\sigma_{r \theta}(r, \pm \pi),
$$

the gap between the crack faces

$$
g=u_{\theta}(r,-\pi)-u_{\theta}(r, \pi),
$$

and the slip velocity

$$
V=\frac{d}{d t}\left[u_{r}(r,-\pi)-u_{r}(r, \pi)\right] .
$$

It should be noted that elasticity problems with friction require that the deformation process be followed in time. We refrain, however, from indicating the time dependence explicitly, unless there is a need to do so. It is assumed in the present discussion that the rate at which the applied force changes in magnitude or direction is sufficiently small, so that inertia effects can be left out.

If the faces of the cut are separated, the boundary conditions are

$$
\begin{aligned}
& N=0, \quad S=0, \\
& \mathrm{~g}>0 .
\end{aligned}
$$

The boundary conditions for contact with stick are

$$
\begin{array}{ll}
g=0, & V=0, \\
N<0, & |S|<f_{s}|N|,
\end{array}
$$

which for contact with slip between the faces

$$
\begin{array}{ll}
g=0, & |S|=f_{k}|N|, \\
N<0, & \operatorname{sgn} S=\operatorname{sgn} V,
\end{array}
$$

while $f_{s}$ and $f_{k}$ denote respectively the coefficients of static and kinetic friction $\left(f_{k} \leq f_{s}\right)$.

The Coulomb law expressed by (8b), (9b) and (10b) makes a distinction between static and kinetic friction. It is generally not possible to incorporate this distinction in elasticity solutions, and one may have to set $f_{k}=f_{s}$. The present problem is an 
exception, because the faces of the cut either stick or slip everywhere. In case the distinction between $f_{k}$ and $f_{s}$ can be maintained, conditions (8b), (9b) and (10b) are not enough to derive unique results, and supplementary requirements must be injected. Thus it is demanded on physical grounds that the change from slip to stick is continuous, whereas a sudden jump in the magnitude (but not sign) of shearing stresses is allowed when stick changes to slip.

The elastic fields suitable for solving the problems considered correspond to the Airy stress function

$$
\begin{aligned}
U=\frac{1}{2 \pi}\left\{A r \theta \sin \theta+B r \theta \cos \theta+\frac{\kappa-1}{\kappa+1}(C r \log r \cos \theta+D r \log r \sin \theta)\right\}, & \\
-\pi<\theta<\pi &
\end{aligned}
$$

in which $A, B, C$ and $D$ are coefficients to be determined and the factor $(\kappa-1) /$ $(\kappa+1)$ is included for convenience. The first two of the unknown coefficients follow immediately from the requirement that all closed contours surrounding the origin transmit the net force $(P \cos \alpha, P \sin \alpha)$. Thus

$$
A=-P \cos \alpha, \quad B=P \sin \alpha .
$$

\section{Separation versus contact}

\section{Separation}

The boundary conditions $(5 a, b)$ yield

$$
C=D=0,
$$

and the gap between the faces of the cut is given by

$$
2 \mu g=\frac{1}{2} P(\kappa-1) \cos \alpha
$$

where $\mu$ is the shear modulus. The inequality (6) restricts the direction of the applied concentrated force to the angles

$$
-\pi \leq \alpha<-\pi / 2 \text { and } \pi / 2<\alpha \leq \pi .
$$

Thus the classical solution is valid only if the concentrated force has a negative $x$-component.

\section{Contact}

The two faces of the cut are in contact for

$$
-\pi / 2<\alpha<\pi / 2 \text {. }
$$


The boundary condition ( $7 \mathrm{a})$ or $(9 \mathrm{a})$ then gives

$$
C=P \cos \alpha,
$$

and, consequently,

$$
\begin{aligned}
& N=-\frac{P}{2 \pi r} \frac{\kappa-1}{\kappa+1} \cos \alpha, \\
& S=\frac{D}{2 \pi r} \frac{\kappa-1}{\kappa+1}, \\
& 4 \mu V=(\kappa-1) \frac{d}{d t}(P \sin \alpha-D) .
\end{aligned}
$$

It is seen from (18) that the inequality (8a) or (10a) is satisfied for all angles $\alpha$ in the range given by (16).

The remaining free coefficient $D$ must be determined from the friction law taking into account the change in the applied force with time.

\section{Force in a fixed direction and with a fluctuating magnitude}

Consider the case when the applied force starts from zero, or

$$
P(0)=0
$$

and acts in a fixed direction. Of interest is only the case with the faces of the cut being in contact, and the angle $\alpha$ can be restricted to the range

$$
0 \leq \alpha<\pi / 2
$$

without loss of generality.

\section{Complete stick}

The boundary condition (7b) together with (20) gives

$$
D=P \sin \alpha
$$

because the integration constant must vanish on account of (21), (18) and (19). The Airy stress function for this case is seen to be identical to that for the unbounded solid without the cut [1]. The inequality (8b) restricts the angle $\alpha$ to

$$
0 \leq \tan \alpha<f_{s}
$$

for complete stick to take place.

The remaining and more interesting range of $\alpha$ is that of $f_{s}<\tan \alpha<\infty$ in which slip occurs. We consider in this range a load that at first increases steadily, next is allowed to decrease and then increases again, as indicated in Fig. 2. 


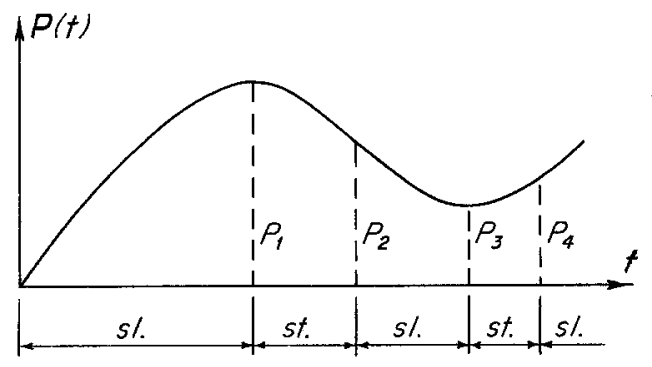

Figure 2

\section{Forward slip upon loading}

The boundary condition (9b) together with (18) and (19) gives

$$
|D|=P f_{k} \cos \alpha \text {. }
$$

The choice of negative value for $D$ violates the condition that the directions of friction stress and slip velocity be consistent. In contrast,

$$
D=P f_{k} \cos \alpha
$$

yields

$$
\begin{aligned}
& S=\frac{P f_{k}}{2 \pi r} \frac{\kappa-1}{\kappa+1} \cos \alpha>0, \\
& 4 \mu V=\dot{P}(\kappa-1) \cos \alpha\left(\tan \alpha-f_{k}\right)>0,
\end{aligned}
$$

and (10b) is satisfied as long as $\dot{P}(t)>0$, or the load is increasing.

\section{Stick upon unloading}

Suppose that the applied concentrated force, having reached the local maximum value $P_{1}$, starts to decrease. It is anticipated that then the faces of the cut stick. The boundary condition (7b) together with (20) gives

$$
\dot{D}=\dot{P} \sin \alpha, \quad D=P \sin \alpha+K .
$$

The integration constant $K$ must be adjusted so that there is no jump in stresses as the conditions change from forward slip to stick. This means that the value of $D$ from (29b) must equal that from (26) for $P=P_{1}$. Thus

$$
D=\left\{P_{1} f_{k}-\left(P_{1}-P\right) \tan \alpha\right\} \cos \alpha .
$$

The stick condition can endure as long as (8b) is not violated, or

$$
\left|P_{1} f_{k}-\left(P_{1}-P\right) \tan \alpha\right|<f_{s} P \text {. }
$$


For $P$ very near $P_{1}$, the quantity inside the absolute value signs is positive, and it can be deduced that the inequality is satisfied. Hence the inequality for a decreasing load can only be violated when this quantity becomes negative, and it follows that stick can persist upon unloading as long as

$$
P>P_{2}=P_{1} \frac{\tan \alpha-f_{k}}{\tan \alpha+f_{s}} .
$$

It is interesting to note that, assuming immediate backslip upon unloading, the conditions $(9 \mathrm{~b})$ and $(10 \mathrm{~b})$ can be satisfied with a value of $D$ that is the negative of that given by (26). However, this value of $D$ gives a jump in stresses, whereas (30) yields a continuous change.

\section{Backslip}

Backslip starts when the magnitude of the applied force falls below the value $P_{2}$ given by (32). For $\dot{P}(t)<0$,

$$
D=-P f_{k} \cos \alpha
$$

The drop in the friction stress when stick changes to backslip is

$$
\Delta S=\frac{P_{1}}{2 \pi r} \frac{\kappa-1}{\kappa+1}\left(f_{s}-f_{k}\right) \frac{\tan \alpha-f_{k}}{\tan \alpha+f_{s}} \cos \alpha .
$$

Stick and subsequent forward slip upon reloading

Suppose that the load is allowed to drop to $P_{3}<P_{2}$, so that backslip is involved, and then the force is increased again. At first, stick sets in, and

$$
D=\left\{\left(P-P_{3}\right) \tan \alpha-P_{3} f_{k}\right\} \cos \alpha
$$

by the same arguments that led to (30). Stick persists as long as

$$
P<P_{4}=P_{3} \frac{\tan \alpha+f_{k}}{\tan \alpha-f_{s}} .
$$

Forward slip starts when $P$ becomes larger than $P_{4}$, and

$$
D=P f_{k} \cos \alpha .
$$

It is interesting to note that $\boldsymbol{P}_{1}$ does not appear in the last three relations, which means that the previous backslip has erased any dependence on the history of the initial forward slip. The implications of this observation for a force fluctuating periodically about a certain level are clear: a steady state is reached in less than one cycle. 


\section{Reloading without previous backslip}

Suppose that the applied force $P$ drops below $P_{1}$, so that stick is established, but is subsequently increased before the backslip condition is reached. The constant $D$ for the stick phase under increasing load is given by (30), and stick prevails as long as (31) is not violated. For increasing load, the inequality becomes critical for a positive value of the quantity inside the absolute value signs, and consequently forward slip commences again when

$$
P>P_{1} \frac{\tan \alpha-f_{k}}{\tan \alpha-f_{s}} .
$$

For the forward slip phase, $D$ is given by (26). The drop in the friction stress as stick changes to slip is

$$
\Delta S=\frac{P_{1}}{2 \pi r} \frac{\kappa-1}{\kappa+1}\left(f_{s}-f_{k}\right) \frac{\tan \alpha-f_{k}}{\tan \alpha-f_{s}} \cos \alpha
$$

which is much larger than going from stick to backslip.

\section{Rotating force with a constant magnitude}

Consider next a rotating force of constant magnitude $P_{0}$. Although the same conclusions can be reached for any monotonically increasing $\alpha(t)$, or $\dot{\alpha}(t)>0$, the results will be written for uniform rotation, or $\alpha=\omega t$. Equations (18) and (19) remain in force, and (20) becomes

$$
4 \mu V=(\kappa-1)\left(P_{0} \omega \cos \alpha-\dot{D}\right) .
$$

It is enough to consider one revolution as the process obviously repeats itself.

For $-\pi \leq \alpha<-\pi / 2$, the faces of the cut are separated, but the gap is closed at $\alpha=-\pi / 2$. It is not immediately clear, however, whether the first phase of contact involves slip or stick. Assuming slip,

$$
D= \pm P_{0} f_{k} \cos \alpha
$$

from $(9 b)$, and

$$
4 \mu V=P_{0} \omega(\kappa-1) \cos \alpha\left(1 \pm f_{k} \tan \alpha\right) .
$$

Choosing the positive sign in (41), $S>0$ and $V<0$, whereas for the negative sign $S<0$ and $V>0$ in the vicinity of $\alpha=-\pi / 2$. Consequently (10b) cannot be satisfied, and the first phase of contact must involve stick.

Starting with stick, (7b) gives

$$
D=P_{0} \sin \alpha+K,
$$

and, since $S=0$ for $\alpha=-\pi / 2$,

$$
D=P_{0}(1+\sin \alpha) \text {. }
$$


The two faces of the cut can stick as long as (8b) is satisfied, or

$$
1+\sin \alpha<f_{\mathrm{s}} \cos \alpha \text {. }
$$

This inequality restricts the angle $\alpha$ to

$$
-\pi / 2<\alpha<\alpha^{*}, \quad \sin \alpha^{*}=-\frac{1-f_{s}^{2}}{1+f_{s}^{2}} .
$$

At $\alpha=\alpha^{*}$, stick changes to slip, and (41) and (42) must be used. Choosing the positive sign in (41), $S>0$, and $V>0$ if

$$
\tan \alpha>-\frac{1}{f_{k}} .
$$

The question is, of course, whether (47) is satisfied for any $\alpha>\alpha^{*}$. From (46b)

$$
\tan \alpha^{*}=-\frac{1-f_{s}^{2}}{2 f_{s}}
$$

and

$$
\frac{1}{f_{k}} \geq \frac{1}{f_{s}}>\frac{1}{f_{s}}-\frac{1+f_{s}^{2}}{2 f_{s}}=\frac{1-f_{s}^{2}}{2 f_{s}} .
$$

Consequently the signs of $S$ and $V$ are the same for all $\alpha>\alpha^{*}$.

The choice of the negative sign in (41) satisfies (10b) for $\tan \alpha>1 / f_{k}$, and it may appear acceptable for sufficiently large $f_{s}$ such that $\left(f_{s}^{2}-1\right) / 2 f_{s}>1 / f_{k}$. However, the change from stick to slip involves a reversal in sign of the friction stresses, and this choice must be discarded as a possibility.

Thus in view of the last four relations, it is clear that positive slip starts at $\alpha=\alpha^{*}$ and persists up to $\alpha=\pi / 2$ when the faces of the cut start to separate.

\section{Conclusion}

The following problems are suitable for student exercises:

1. Force with a constant magnitude $P_{0}$ and oscillating direction $\left(\alpha=\alpha_{0} \sin \omega t\right)$.

2. Force with a constant $x$-component $P_{x}$, and a fluctuating $y$-component $P_{y}$ possibly involving sign reversals.

\section{REFERENCES}

[1] Timoshenko, S. and Goodier, J. N., Theory of elasticity, 3rd ed. McGraw-Hill 1970.

[2] Dundurs, J. and Stippes, M., Role of elastic constants in certain contact problems. Journal of Applied Mechanics, 37 (1970) 965-970. 